\title{
Parameter Estimation Using Ensemble-Based Data Assimilation in the Presence of Model Error
}

\author{
JUAN RUIZ \\ Centro de Investigaciones del Mar y la Atmósfera (CIMA/CONICET-UBA), DCAO/FCEyN-Universidad de Buenos \\ Aires, UMI-IFAECI/CNRS, Buenos Aires, Argentina, and AICS/RIKEN, Kobe, Japan \\ MANUEL PULIDO \\ Department of Physics, Universidad Nacional del Nordeste, IMIT (UNNE-CONICET), Corrientes, \\ and UMI-IFAECI/CNRS, Buenos Aires, Argentina
}

(Manuscript received 21 December 2013, in final form 22 October 2014)

\begin{abstract}
This work explores the potential of online parameter estimation as a technique for model error treatment under an imperfect model scenario, in an ensemble-based data assimilation system, using a simple atmospheric general circulation model, and an observing system simulation experiment (OSSE) approach. Model error is introduced in the imperfect model scenario by changing the value of the parameters associated with different schemes. The parameters of the moist convection scheme are the only ones to be estimated in the data assimilation system. In this work, parameter estimation is compared and combined with techniques that account for the lack of ensemble spread and for the systematic model error. The OSSEs show that when parameter estimation is combined with model error treatment techniques, multiplicative and additive inflation or a bias correction technique, parameter estimation produces a further improvement of analysis quality and mediumrange forecast skill with respect to the OSSEs with model error treatment techniques without parameter estimation. The improvement produced by parameter estimation is mainly a consequence of the optimization of the parameter values. The estimated parameters do not converge to the value used to generate the observations in the imperfect model scenario; however, the analysis error is reduced and the forecast skill is improved.
\end{abstract}

\section{Introduction}

Parameter estimation using data assimilation is an objective and efficient methodology that might be used to optimize uncertain parameters in numerical models. In this methodology, model parameters are treated as state variables (Jazwinski 1970) so they can be optimized based on the available observations. Several recent works discuss parameter estimation using data assimilation techniques (Annan et al. 2005; Aksoy et al. 2006a; Pulido and Thuburn 2006; Kondrashov et al. 2008; Jung et al. 2010; Kang et al. 2011; Bellsky et al. 2014; Zhang et al. 2012; Wu et al. 2012; Ruiz et al. 2013a) among many others. Aksoy (2015) presented a review about the estimation of model parameters using data

Corresponding author address: Juan Ruiz, CIMA (CONICETUniversidad de Buenos Aires), Ciudad Universitaria, Buenos Aires, CABA C1428EGA, Argentina.

E-mail: jruiz@cima.fcen.uba.ar assimilation techniques while Ruiz et al. (2013a) presented a review of parameter estimation using ensemblebased data assimilation techniques as well as some results of its implementation with a simple atmospheric general circulation model.

Most experiments on parameter estimation have been performed under the perfect model assumption (e.g., Aksoy et al. 2006a,b; Koyama and Watanabe 2010; Kang et al. 2011; Ruiz et al. 2013a,b). In these so-called twin experiments, synthetic observations are generated with the model using a certain set of parameters, say true parameters. Then, an almost perfect version of the model, which only differs from the perfect model in the values of the parameters to be estimated is used to simulate a data assimilation cycle. In this idealized situation, the estimated model parameters usually converge to their true values and model error is almost eliminated. This produces a significant improvement in the analysis and also in the short, mediumrange, and long-term forecasts (Koyama and Watanabe 2010; Schirber et al. 2013; Wu et al. 2012; Ruiz et al. 2013a). 
Atmospheric general circulation models contain many sources of model error in realistic applications (e.g., truncation errors and simplifications made in parameterizations; Zhang et al. 2012). These sources of errors cannot be completely eliminated by tuning some model parameters. In parameter estimations for realistic applications, there are no true values for the model parameters, only optimal values for the parameters can be defined that are the ones that maximize the short-range forecast skill or that produce the best representation of the climatology under a certain metric (Annan 2005). The selection of the metric is particularly important since the optimal values for the parameters may depend on this metric. Schirber et al. (2013) performed experiments using an atmospheric general circulation model in which the convective scheme parameters are estimated using an ensemble-based data assimilation technique. They conducted perfect model experiments as well as imperfect model experiments and show that the estimated parameters in the imperfect model experiment are different from the ones obtained in the perfect model experiments. Systematic errors in the observations or in the observation operator may also affect the estimated parameter values. Jung et al. (2010) show, using an observing system simulation experiment and a mesoscale model, that when the observation operator is imperfect the estimated parameters do not converge to their nature values; however, the optimization of some model parameters produces an improvement upon the analysis and the forecast. The success of the optimization depends upon the observations used.

Ensemble-based data assimilation systems are particularly sensitive to model imperfection (Miyoshi 2005; Li et al. 2009; Whitaker et al. 2008). This may be because ensemble methods restrict the corrections introduced by the observations to the subspace spanned by the ensemble members. When the ensemble is not explicitly designed to account for the presence of model errors, the structure of the ensemble forecast perturbations may significantly differ from the structure of model errors and, thus, observations are not able to properly correct them. There are several techniques for considering the effect of model error in data assimilation and in particular for ensemble methods. These techniques might be classified in two categories: The first category is the techniques that deal with the underestimation of the background error covariance due to model error and limited ensemble size. This is particularly the case in weather forecast models whose model state has $10^{8}$ or more dimensions while a few tens or a few hundred ensemble state members can be afforded in the data assimilation system. The model error techniques that fall into this category, such as additive inflation (Houtekamer et al. 2009; Li et al. 2009), adaptive multiplicative inflation (Miyoshi 2011; Anderson 2009), multimodel ensembles (Meng and Zhang 2007), stochastic kinetic energy backscatter (Shutts 2005), stochastically perturbed parameterization tendencies (Buizza et al. 1999), and parameter perturbations (Jung et al. 2010; Stainforth et al. 2005) attempt to consider the missing sources of uncertainty in the estimation of the background error covariance. They do not attempt to alleviate the model error. The second category is those techniques that estimate the systematic model error and partially correct it (i.e., bias correction methods) (Dee and da Silva 1998; Baek et al. 2006; Danforth et al. 2007). Within the framework of ensemble-based data assimilation, parameter estimation may be used for model error treatment. Online parameter estimation belongs to both categories because it may alleviate systematic model errors by optimizing incorrect parameter values. But also, it can contribute to enhance the model ensemble spread because in order to estimate covariances between the parameters and the observations each ensemble member uses a different set of model parameters (Aksoy 2015).

The combination of parameter estimation with other methods that take into account model error in a data assimilation system has not been explored yet. In this work, we evaluate online parameter estimation as a model error treatment technique combined with other state-of-the-art techniques for dealing with model error. In particular, we examine whether parameter estimation can provide a further improvement in the analysis beyond the improvement produced by the other techniques for model error treatment. The fact that, online parameter estimation may contribute to both ensemble spread enhancement and to correct systematic model error, makes it particularly suitable for these combined experiments. The techniques are evaluated using a simple atmospheric general circulation model in experiments with observations taken from model integrations. To produce the synthetic observations, the model integration uses a large set of parameters that are changed with respect to the model used in the data assimilation system, including parameters related to the schemes of vertical diffusion, large-scale condensation, deep moist convection, and surface exchange of heat and momentum from land and sea. The parameters of the moist convection scheme are the only ones to be estimated in the data assimilation cycle. In this way, the model has errors in reproducing several physical processes that cannot be directly corrected, by parameter optimization, in the data assimilation cycle except for moist convection. The data assimilation experiments evaluate the impact of parameter estimation and its combination with other 
model error techniques upon the analysis and mediumrange forecasts.

This paper is organized as follows: section 2 describes the methodology and data used in this work, section 3 shows the results, and in section 4 we draw the conclusions.

\section{Methodology}

\section{a. Model}

In this work, a simplified and computationally efficient atmospheric general circulation model known as the Simplified Parameterizations, Primitive Equation Dynamics (SPEEDY) model is used (Molteni 2003). This model has a T42 spectral representation of the atmospheric fields and seven vertical sigma levels. The model includes some simple parameterizations of convection, radiation, large-scale condensation, planetary boundary layer, and surface processes. There is also a simple but realistic representation of the land-sea distribution. This model is extensively used for idealized data assimilation experiments given its computational efficiency (Miyoshi 2005; Fertig et al. 2007; Kang et al. 2011).

\section{b. Data assimilation algorithm}

The ensemble-based data assimilation algorithm implemented in this work is similar to the one described in Miyoshi and Yamane (2007). This data assimilation algorithm is based on the local ensemble transform Kalman filter (LETKF) introduced by Hunt et al. (2007). In the LETKF, the Kalman filter equations are solved in the subspace spanned by the forecast ensemble perturbations, which makes the computation more efficient and easier to parallelize.

A common practice in ensemble Kalman filter methods is to apply some kind of localization to reduce the impact of sampling error in the estimation of error covariances from a limited size ensemble, thus increasing the rank of the estimated background error covariance matrix (Hamill et al. 2001). In the current implementation, localization is achieved applying an observation error covariance localization approach (Greybush et al. 2011; Miyoshi et al. 2007).

An ensemble of 50 members is used in all the experiments discussed in this work. The ensemble state members are initialized taking random samples from the SPEEDY model climatology so no information about the current state of the system is included at the beginning of the assimilation cycle.

\section{c. Model error treatment}

In this section a brief description of the different model error techniques used in this paper is provided.

\section{1) Multiplicative inflation}

Multiplicative inflation consists on multiplying the ensemble perturbations by an inflation parameter (Anderson 2001), which can be time and space dependent (Anderson 2009; Miyoshi 2011). Even when model error may be considered negligible, multiplicative inflation can reduce the impact of sampling error that usually occurs when lowrank estimations of the error covariance matrices are used. In the presence of model error, multiplicative inflation can also be used to increase the forecast ensemble spread as a way to account for model error ( $\mathrm{Li}$ et al. 2009; Miyoshi 2011). In this work, the multiplicative inflation factor $\delta_{m}$ is assumed to be constant in space and time. An optimal value of 1.125 has been determined based on tuning experiments ( $\mathrm{Li}$ et al. 2009).

\section{2) AdDitive InFLAtion}

Additive inflation consists of adding different perturbations to each ensemble member. This method has been found to produce a better representation of model errors when compared with multiplicative inflation (Whitaker and Hamill 2012; Li et al. 2009; Whitaker et al. 2008). Ideally, the additive ensemble perturbations should represent possible realizations of the model error. This is particularly difficult to achieve since model error is usually unknown. Following Li et al. (2009), in this work, additive perturbations are generated as differences between the 6-h forecast produced with the perfect model and with the imperfect model. Thus, the perturbations are taken from true possible realizations of model error. At each assimilation step, a set of 50 additive perturbations is randomly selected from a large sample (over 5000) of model error realizations and added to the ensemble members after the analysis and before the model integration. The mean of the selected additive perturbations is removed in order to preserve the analysis ensemble mean ( $\mathrm{Li}$ et al. 2009). The amplitude of these perturbations is scaled by a parameter $\delta_{a}$ that in this work is considered constant in space and time. An optimal value of 1.25 has been determined based on tuning experiments ( $\mathrm{Li}$ et al. 2009).

\section{3) BIAS CORRECTION}

Bias correction provides a way to estimate the systematic component of model error within a data assimilation cycle. Several techniques have been developed to infer and remove the model bias (Dee and da Silva 1998; Baek et al. 2006; Li et al. 2009; Greybush et al. 2012; Danforth et al. 2007 among many others). In this work, a bias correction technique, in which the bias is estimated based on the analysis increment ( $\mathrm{Li}$ et al. 2009), is used following the equation below: 


$$
\overline{\mathbf{q}}^{a}=\mu_{b} \overline{\mathbf{q}}^{f}+\delta_{b}\left(\overline{\mathbf{x}}^{f}-\overline{\mathbf{q}}^{f}-\overline{\mathbf{x}}^{a}\right),
$$

where $\overline{\mathbf{q}}^{a}$ is the estimated model bias for the current analysis cycle, $\overline{\mathbf{q}}^{f}$ is the bias estimated for the previous analysis cycle, $\delta_{b}$ is a scaling coefficient, and $\mu_{b}$ is a factor $(<1)$ that relaxes the bias toward 0 . The estimated bias in the previous assimilation cycle, $\overline{\mathbf{q}}^{f}$, is subtracted from each forecast ensemble member before assimilating the observations. An optimal value of 0.1 for $\delta_{b}$ has been determined through tuning experiments; the parameter $\mu_{b}$ has been set to 0.9 following the recommendations of Li et al. (2009).

\section{4) PARAMETER ESTIMATION}

In this work, a state augmentation approach is used in which the parameters to be estimated are included into the state vector (Jazwinski 1970; Cornick et al. 2009). Localization is not applied for the estimation of model parameters because they are global parameters and, therefore, their values can be correlated with model state variables errors at any location (Fertig et al. 2007; Ruiz et al. 2013b). The model parameters to be estimated are initialized with random realizations from a Gaussian distribution with mean parameter values that represent a priori physical estimates of their values, and a standard deviation thath represents an a priori estimate of the uncertainty associated with that parameter (Aksoy et al. 2006a).

In some cases the values of estimated model parameters are subject to physical constraints that are inherent to their definition or purpose (e.g., positive definite parameters). Ensemble Kalman filters assume that the error distribution of the parameters is Gaussian so in principle no constraint is assumed for the estimated parameter values (Aksoy 2015). To prevent the estimated parameters from taking unrealistic values, each individual member of the parameter ensemble is examined after the data assimilation and if one of them is outside the a priori defined range, it is replaced by a random parameter value taken from a Gaussian distribution with mean equal to the parameter ensemble mean and standard deviation equal to the ensemble parameter spread. This procedure is repeated until all the parameter ensemble members are within the a priori defined range.

Persistence is assumed for the evolution of parameter values between two assimilations of observations. Because of this, the parameter ensemble spread is systematically reduced during the data assimilation eventually producing filter divergence for the parameters (Aksoy 2015; Ruiz et al. 2013b). In this work, the approach introduced by Ruiz et al. (2013b) that provides an online
TABLE 1. Values of the parameters used in the imperfect version of the model and in the nature integration.

\begin{tabular}{lcc}
\hline \hline \multicolumn{1}{c}{ Name } & Nature value & Imperfect value \\
\hline TRLSC & 4.0 & 3.0 \\
RHLSC & 0.9 & 0.8 \\
TRSHC & 6.0 & 7.0 \\
TRVDI & 20.0 & 24.0 \\
FWIND0 & 0.6 & 0.5 \\
CDL & $2.2 \times 10^{-3}$ & $2.4 \times 10^{-3}$ \\
CDS & $0.8 \times 10^{-3}$ & $0.7 \times 10^{-3}$ \\
CHL & $1.2 \times 10^{-3}$ & $1.15 \times 10^{-3}$ \\
CHS & $0.8 \times 10^{-3}$ & $0.75 \times 10^{-3}$ \\
TRCNV & 0.16 & 0.25 \\
RHBL & 0.9 & 0.8 \\
ENTMAX & 0.5 & 0.3 \\
\hline
\end{tabular}

estimation of the parameter ensemble spread is used. The impact of this approach upon the estimated parameters and the analysis quality is discussed in section $3 \mathrm{~d}$.

\section{d. Experimental design}

In this work, an observing system simulation experiment approach is used. A 6-month "nature" integration is generated with the general circulation model. Observed variables are assumed to be temperature, zonal and meridional wind, specific moisture, and surface pressure. They are taken every $6 \mathrm{~h}$ from the nature integration, by adding a Gaussian random error with standard deviations of $1 \mathrm{~K}$ for the temperature, $1 \mathrm{~m} \mathrm{~s}^{-1}$ for the wind components, $1 \mathrm{~g} \mathrm{~kg}^{-1}$ for the specific moisture, and $1 \mathrm{hPa}$ for the surface pressure as in Li et al. (2009). The horizontal location of the observations is chosen at each data assimilation cycle using randomly generated longitudes and latitudes with uniform distribution. This is used in order to avoid effects associated with fixed inhomogeneous networks that require more sophisticated schemes for the treatment of inflation (Miyoshi 2011; Whitaker and Hamill 2012). The number of observation locations in each cycle is 1152 and is kept constant in time. At each horizontal location temperature, horizontal wind components and specific humidity observations are generated in all the model levels.

For the data assimilation system experiments, an imperfect version of the SPEEDY model is used (unless otherwise indicated) that is different from the version used to generate the nature integration. The nature integration of the SPEEDY used to produce the observations uses the set of standard parameters of the model shown in Table 1, which are referred to as nature parameters. The imperfect version of the SPEEDY model uses another set of parameters, which are referred as imperfect parameters, and are specified in Table 1. These parameters affect vertical diffusion (TRSCH and TRVDI), large-scale condensation (TRLSC and RHLSC), deep moist convection (TRCNV, 
RHBL, and ENTMAX), and surface exchange of momentum and heat for land (CDL and CHL) and sea (CDS and CHS). When the imperfect version of the model is used in a data assimilation cycle, the resulting analysis error is more than 3 times greater than the one obtained with the perfect model. The parameters of the deep moist convection scheme are the only ones being estimated (TRCNV, RHBL, and ENTMAX) in the parameter estimation experiments. The other parameters whose values have been modified represent sources of model error that cannot be directly accounted for the estimation of deep moist convection parameters.

In this work, the error in the model state is quantified, using the total RMSE defined as

$$
\mathrm{RMSE}=\sqrt{\frac{1}{N}\left(\overline{\mathbf{x}}^{a}-\mathbf{x}^{t}\right)^{\mathrm{T}} \mathbf{A}^{-1}\left(\overline{\mathbf{x}}^{a}-\mathbf{x}^{t}\right),}
$$

where $\overline{\mathbf{x}}^{a}$ is the analysis state vector, $\mathbf{x}^{t}$ is the true state vector, $N$ is the number of state variables, and $\mathbf{A}$ is an $N \times$ $N$ diagonal matrix that normalizes the error by the typical error magnitude for each variable. These typical errors are the same as the ones used to generate the observations.

The systematic component of the error is computed using the total absolute mean error (AME), defined as

$$
\mathrm{AME}=\frac{1}{N} \sum_{n=1}^{N} \mathbf{A}(n, n)^{-1 / 2}\left|\left\langle\overline{\mathbf{x}}^{a}(n)-\mathbf{x}^{t}(n)\right\rangle\right|,
$$

where $\mathbf{x}(n)$ denotes the $n$th component of $\mathbf{x},||$ denotes the absolute value, and \langle\rangle denotes time averaging. Note that to compute AME, first the time average of the error or bias is computed, then its absolute value is taken and finally the sum over different locations and different variables normalized by their typical error magnitude is conducted. Taking the absolute values avoids compensation from biases coming from different regions or variables. Both RMSE and AME are computed considering the entire state vector or for a particular variable.

\section{Results}

\section{a. Parameter estimation as a model error treatment technique}

Four experiments were conducted to evaluate the performance of parameter estimation as a model error treatment technique in comparison with multiplicative and additive inflation. The experiments are (i) multiplicative inflation alone, (ii) multiplicative and additive inflation, (iii) parameter estimation and multiplicative inflation, and (iv) parameter estimation combined with multiplicative and additive inflation. In the parameter estimation experiments, the initial parameter ensemble is generated randomly sampling parameter values from a normal distribution. The mean of the normal distribution is 0.5 for TRCNV, 0.8 for RHBL, and 0.3 for ENTMAX, the standard deviation is the same for all the parameters and equal to $6.1 \times 10^{-3}$. The chosen mean values for the parameter estimation experiments are farther from the nature parameter values than the values used in the imperfect model. In all the experiments shown in this work, time average quantities are computed excluding the first 200 data assimilation cycles to avoid contamination of the diagnostics with the spinup of the filter. In what follows, the experiment that combines multiplicative and additive inflation is used as a reference for comparison and referred as the control experiment.

The time evolution of the total analysis RMSE for the above-mentioned experiments is shown in Fig. 1, the average RMSE for each experiment is also provided. The experiment with additive and multiplicative inflation and the experiment with multiplicative inflation and parameter estimation both have a significant improvement of the analysis quality with respect to the experiment with multiplicative inflation alone. When parameter estimation is added to multiplicative and additive inflation, there is a further important reduction in the analysis RMSE.

The reason for the improvement produced by parameter estimation in the analysis RMSE may be traced back to two possible explanations. One explanation is that the spread of the parameters introduces more spread into model state variables that produces a better representation of the model state uncertainty. A second explanation may be that parameter estimation produces a convergence of the parameter ensemble mean values toward optimal values correcting systematic model errors and, therefore, improving the analysis quality. The values of the time-averaged estimated parameters in the parameter estimation experiment with multiplicative and additive inflation are 0.50 for TRCNV, 0.77 for RHBL, and 0.80 for ENTMAX, which differ from the nature values (see Table 1 ).

To evaluate the impact of estimated parameter values upon the analysis quality, a second set of two data assimilation experiments were performed. One experiment in which the convective scheme parameters are set to the nature values (see Table 1) and a second experiment, where the convective scheme parameters are set to a time average of the estimated parameters obtained in the parameter estimation experiment. Both experiments use multiplicative and additive inflation. Figure 2 shows the evolution of the analysis RMSE for these two experiments in comparison with the RMSE from the 


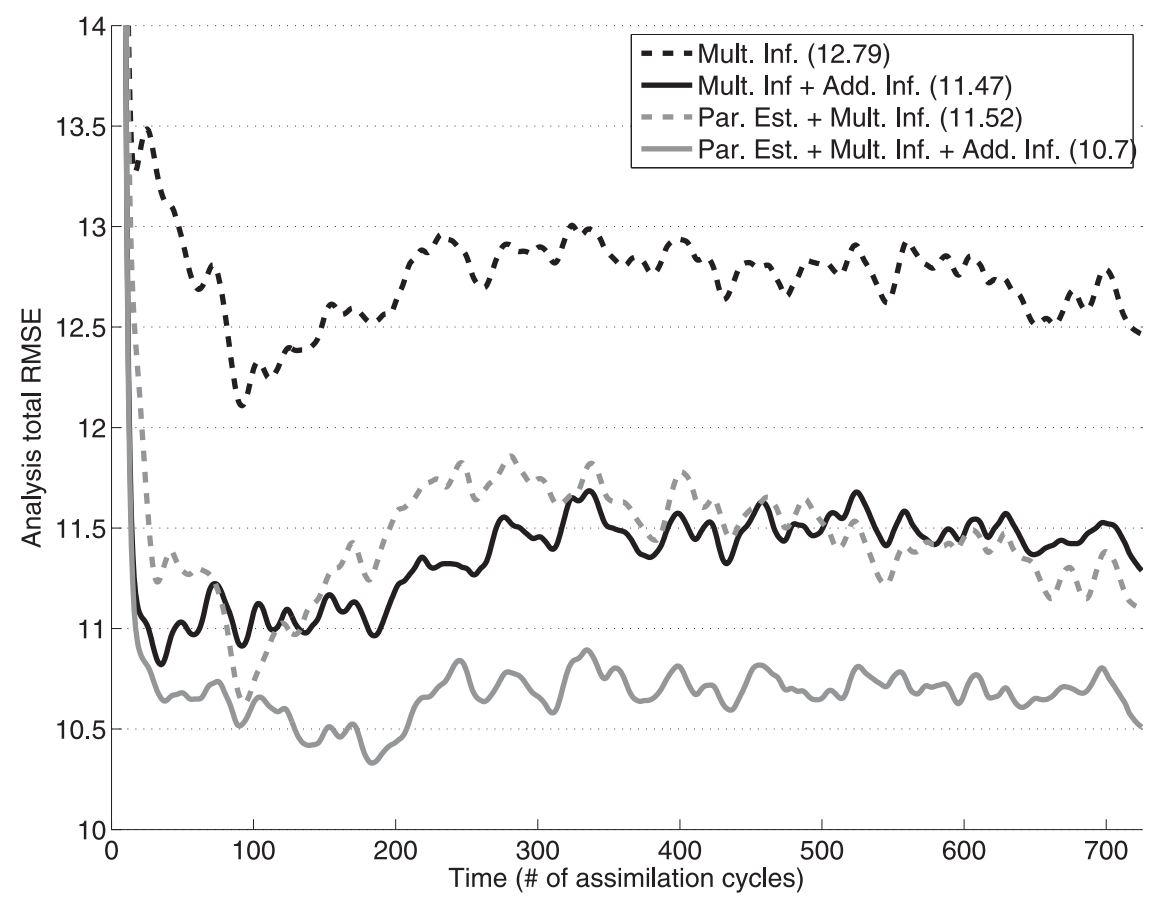

FIG. 1. Total analysis RMSE as a function of time for the experiment with multiplicative inflation alone (black dashed line), multiplicative and additive inflation (black solid line), parameter estimation with multiplicative inflation (gray dashed line), and parameter estimation with multiplicative and additive inflation (gray solid line) experiments. The average RMSE for each experiment is shown between brackets in the legend.

control experiment and the parameter estimation experiment. The experiment that uses the nature convective scheme parameters has almost the same RMSE as the control experiment (that uses an imperfect set of convective scheme parameters). On the other hand, the experiment with the time-averaged estimated parameters leads to a $6.7 \%$ reduction in the RMSE with respect to the experiment performed with the nature convective scheme parameters and also with respect to the control experiment. The optimal parameters in the imperfect model are able to reduce the analysis RMSE with respect to the nature parameters. These experiments show that the correction of the systematic model error produced by optimal parameters has an important impact in the analysis RMSE. This result also suggests that the optimization of the convective scheme may be canceling out errors in the model coming from other parameterizations with imperfect parameters. Therefore, although the estimated convective scheme parameters are not the nature values, they minimize the total analysis RMSE.

The only difference between the experiment with time-averaged estimated parameters and the parameter estimation experiment is that in the parameter estimation experiment, the parameter values can change in time as a consequence of the estimation process. Figure 2 shows that the experiment with time-averaged estimated parameters is as good as the parameter estimation experiment. This suggests that time fluctuations in the estimated parameters are mostly associated with noise in the estimated parameters and do not contribute significantly to the improvement of the analysis quality. The origin of these fluctuations are mostly associated with sampling error and with time-dependent projections of model error in the direction of model sensitivity to the parameters [see Aksoy (2015) and Tong and Xue (2008) for further discussion on this issue and for approaches to reduce the impact of sampling error upon the estimated parameters].

The impact of the spread of the parameters produced by parameter estimation on the analysis RMSE is examined by means of an experiment in which the convective parameters are not estimated, but they are Gaussianly distributed around its assumed fixed mean value that is set to the imperfect model parameter values (see Table 1). The spread of the parameters is the time average of the parameter ensemble spread estimated in the parameter estimation experiment for each individual parameter. Figure 3a shows that the experiment with perturbed parameters has a similar analysis RMSE that the control experiment. This shows that the perturbations introduced in the parameters produce a negligible impact upon the analysis quality and almost all of the improvement in the 


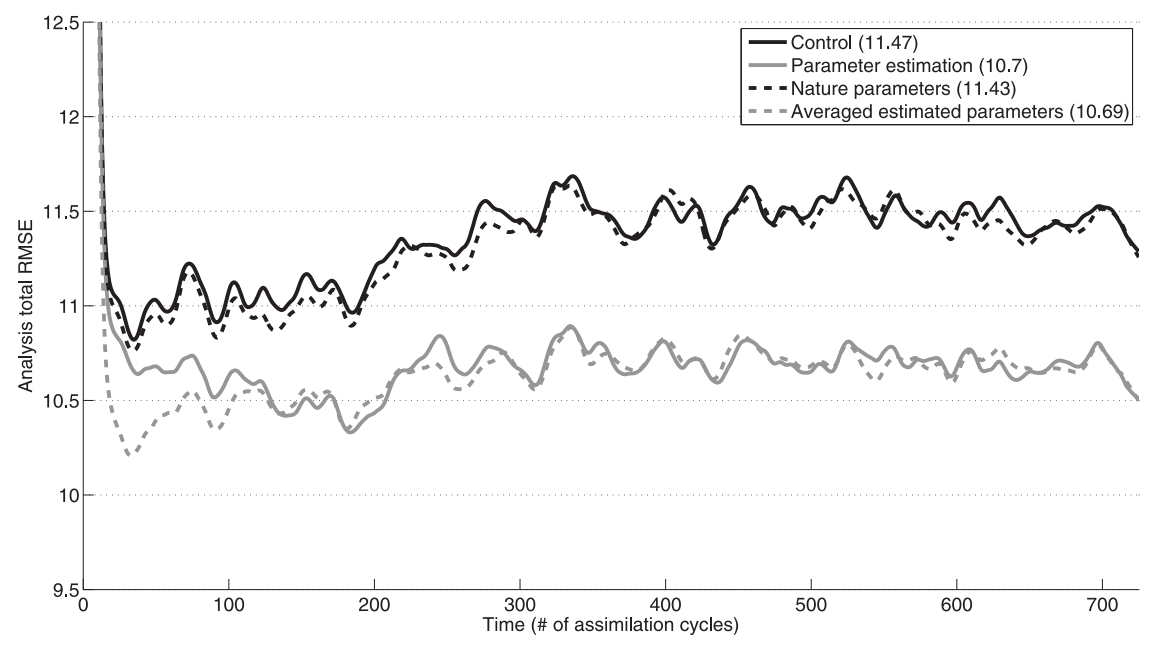

FIG. 2. Total analysis RMSE as a function of time for the control experiment (black solid line), parameter estimation (gray solid line), nature convective scheme parameters (black dashed line), and time-averaged estimated convective scheme parameters (gray dashed line) experiments.

parameter estimation experiment comes from the update of the parameter value.

The correction of systematic errors by online parameter estimation appears to have a large impact in the analysis RMSE. Two additional experiments explore the impact of correction of systematic errors: one in which a bias correction technique is applied and the other uses a combination of bias correction and parameter estimation. Both experiments use multiplicative and additive inflation. The bias correction experiment gives a RMSE smaller than the experiment that uses only multiplicative and additive inflation (Fig. 4). The improvement produced by the bias correction in this case is close to the one produced by the parameter estimation. Further improvement is obtained when bias correction and parameter estimation techniques are combined. As can be seen in Fig. 4, the impact of estimating the convective scheme parameters is smaller if a bias correction method is implemented in the data assimilation cycle. Bias correction can partially correct the systematic component of model error, in particular the one associated with nonoptimal model parameters, thus reducing the additional improvement produced by parameter estimation. Even though, adding parameter estimation can improve the analysis RMSE given by the bias correction method.

\section{b. Impact of estimated parameters upon individual variables}

Figure 5 shows the vertical profile of the analysis AME [Eq. (3)] for zonal and meridional wind, temperature, and specific humidity for the control, parameter estimation, and bias correction experiments. The impact of parameter estimation is stronger for $U$ and $V$ wind components at mid- and upper levels and for temperature at upper levels. This impact does not seem to be directly related to the model sensitivity to the convective scheme parameters. The sensitivity to these parameters is stronger for temperature at midlevels and for wind at lower and upper levels as shown in Ruiz et al. (2013a). The bias correction experiment reduces the systematic component of the analysis error for all model variables, including specific moisture, with respect to the control experiment.

As shown in Fig. 5, the analysis AME for specific moisture is degraded in the parameter estimation experiment with respect to the control experiment. This is an unexpected result since low-level moisture is directly associated with convection, so that an optimization of convective scheme parameters was expected to improve the representation of low-level moisture. One possible explanation is that the optimal parameter values that are different from the nature values are forcing the convective scheme to produce improvements in all model variables but specific moisture. In particular, the optimal TRCNV parameter in the parameter estimation experiment is larger than the nature value. This leads to a faster adjustment time in the convective scheme. The estimated optimal RHBL parameter in this experiment is smaller than the nature value, relaxing low-level specific moisture to a lower value in the planetary boundary layer. The combination of these two effects leads to an increase in the strength of convection in the model, thus reducing moisture and producing a dry bias. As a result, the spatially averaged lowest-level moisture bias in the parameter estimation experiment is $-0.47 \mathrm{~g} \mathrm{~kg}^{-1}$ while it is $-0.43 \mathrm{~g} \mathrm{~kg}^{-1}$ in the control experiment. This shows how convection and the hydrological cycle are affected through 


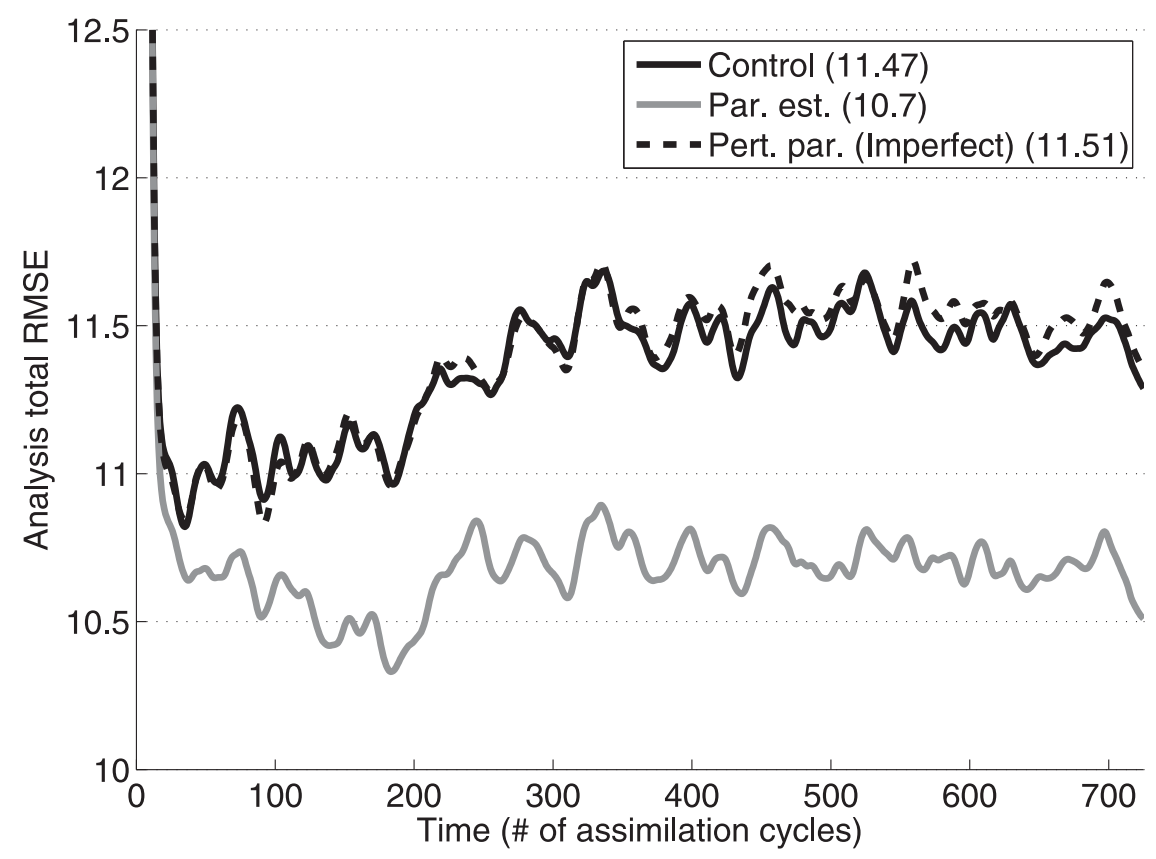

FIG. 3. Total analysis RMSE as a function of time for the control (black solid line), parameter estimation (gray solid line), and perturbed parameter (black dashed line) experiments.

parameter estimation to partially correct other errors in the model.

The degradation in low-level moisture in the parameter estimation experiment, suggests that the parameter values that improve low-level moisture forecasts are different from those that improve wind and temperature forecasts. To evaluate this hypothesis, we conducted an extreme experiment in which the parameters are only being estimated from specific moisture observations at low levels (below $700 \mathrm{hPa}$ ). All available observations are used for the state variable assimilation as in the previous experiments. Figure 5 shows that parameter estimation using only specific moisture observations reduces the analysis AME for this variable at low levels in which specific moisture is observed. On the other hand, it degrades the AME for all the other model variables including specific moisture at upper levels. This shows that the estimated parameters are sensitive to the observations used in the optimization (Kang et al. 2011; Schirber et al. 2013). The estimated parameters in this experiment repeatedly adopt nonphysical values (not shown). The tendency of the model parameters to drift away from the physically meaningful range in this experiment also highlights the need for further evaluation of parameter estimation with real observations for which constraining the parameter to an expert-defined range would be essential for the success of the technique.

When parameter estimation is used to correct model error, the correction is done in a space that usually has a few dimensions (three in this work which is the number of the estimated parameters), then the correction in different model variables is linked through the model sensitivity to the parameters that are being estimated. This can lead to the type of results discussed in this section in which some variables are improved at the expenses of a degradation in some other variable.

\section{c. Impact of estimated parameters upon the medium-range forecasts}

An ensemble of 50 medium-range forecasts are generated to a maximum lead time of 10 days using the initial conditions from the control, parameter estimation, bias correction, and the combined parameter estimation and bias correction data assimilation experiments. In the bias correction experiment, the estimated bias in the analysis cycle is also used to correct the model tendencies in the forecast every $6 \mathrm{~h}$ as in Danforth et al. (2007). This is done in order to partially correct the model error, not only in the initial condition, but also in the forecast. In the parameter estimation experiment, the estimated parameter ensemble members (i.e., the analysis parameter ensemble) corresponding to the forecast initialization time are used to produce the forecast. In that way, the forecast ensemble members include perturbations in the initial conditions and in the model parameters.

Figure 6a shows the total RMSE of the ensemble mean as a function of the forecast lead time. The relative differences in the RMSE among the forecast experiments 


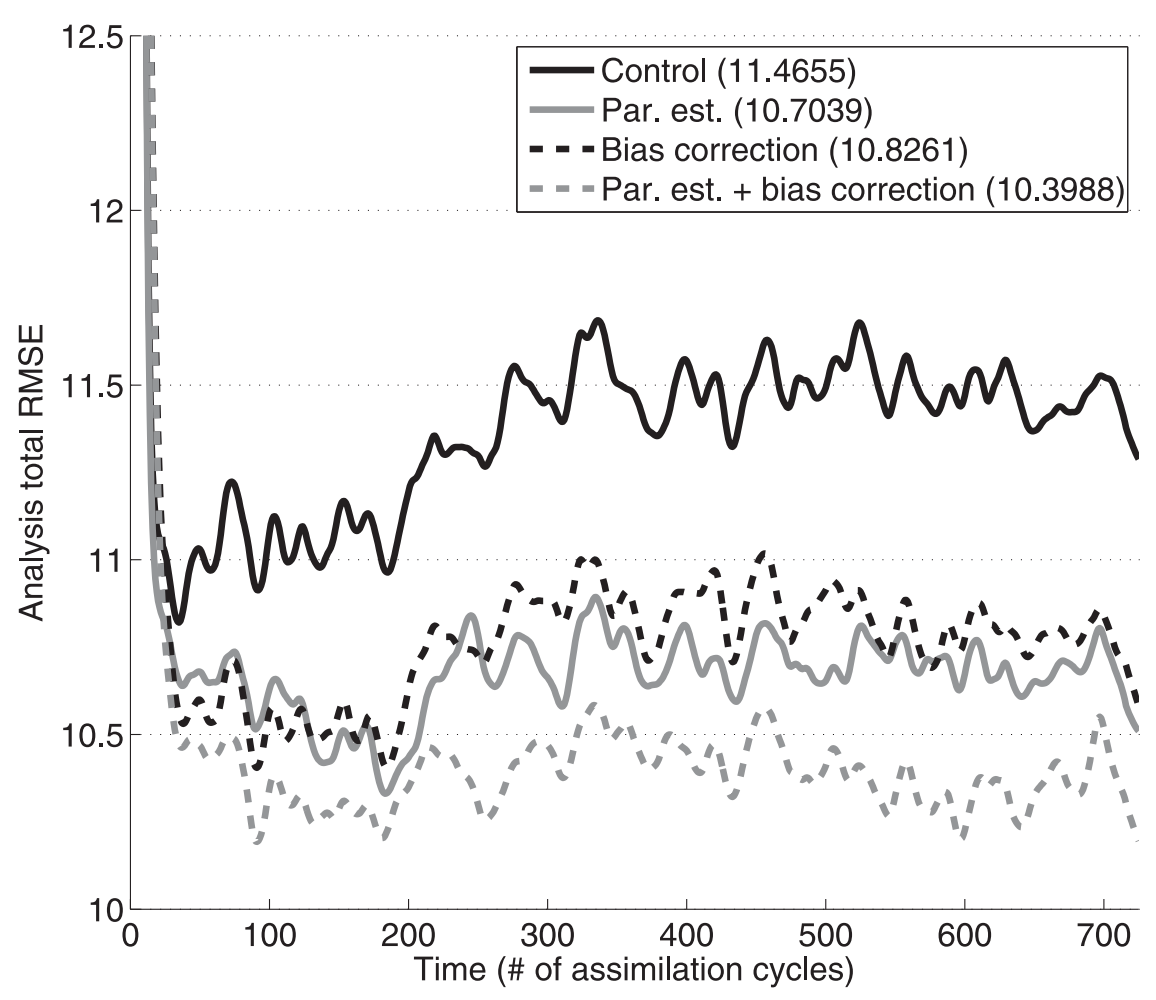

FIG. 4. Total analysis RMSE as a function of time for the control (black solid line), parameter estimation (gray solid line), bias correction (black dashed line), and combination of parameter estimation with bias correction (gray dashed line) experiments.

are basically the same as those found in the analysis (section 3a). The combination of bias correction and parameter estimation is the experiment that produces the lowest forecast RMSE, followed by parameter estimation without bias correction. For most variables, the relative impact of parameter estimation grows with time, indicating an improvement due to better initial conditions (i.e., more accurate analysis), but also due to parameter values that reduce model error during the forecast. In these experiments, the improvement of the forecast quality produced by parameter estimation with respect to the control forecast quality is larger than the improvement produced by the bias correction scheme. This shows that, in these idealized experiments, even though the estimated parameters do not converge to the nature parameters, the estimated parameter values improve the forecast up to 10 days of lead time (near the predictability limit for synoptic-scale flow). The positive impact of parameter estimation in the forecasts is found in all model variables, even for specific moisture (Fig. 6c). Specific moisture forecasts are worse in the parameter estimation experiments, for the shorter lead times (less than $24 \mathrm{~h}$ ), but for longer lead times, parameter estimation experiments produce a better representation of the moisture field. One possible explanation for the lower moisture
RMSE at longer forecast ranges found for the parameter estimation experiment is that the improvement in the representation of other model variables, like the wind field, produces a positive impact upon the quality of the forecasted moisture field through a better representation of the moisture advection.

\section{d. Sensitivity of parameter estimation to initial parameter ensemble mean and spread}

In this work, two approaches for the determination of the parameter ensemble spread are examined: the conditional covariance inflation (CCI) approach (Aksoy et al. 2006a), which keeps the spread of each estimated parameter constant in time, and the estimated parameter ensemble spread (EPES) approach (Ruiz et al. 2013b), which provides an online estimation of the parameter ensemble spread. The EPES approach is the one used in the experiments shown in previous sections. Both approaches use a time-dependent inflation coefficient for the parameter ensemble spread that is independent of the inflation coefficient used for the model state variables. The two approaches are compared in the perfect model scenario in Ruiz et al. (2013b). In that work, it is shown that the EPES approach allows an online estimation of the optimal value for the parameter 
(a) $-U$

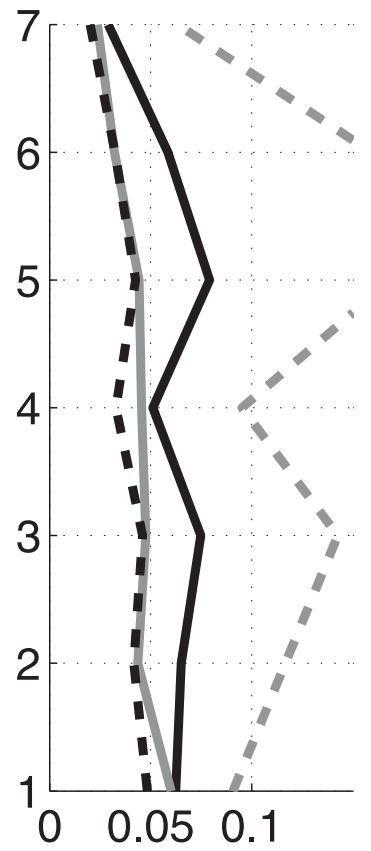

(b) $-\mathrm{V}$

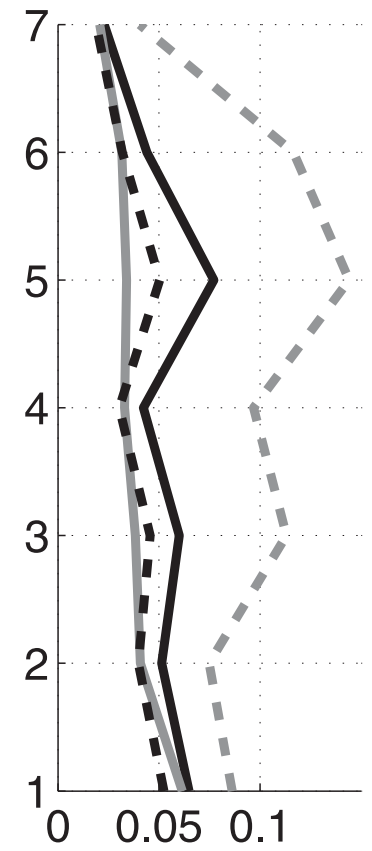

(c) $-\mathrm{T}$

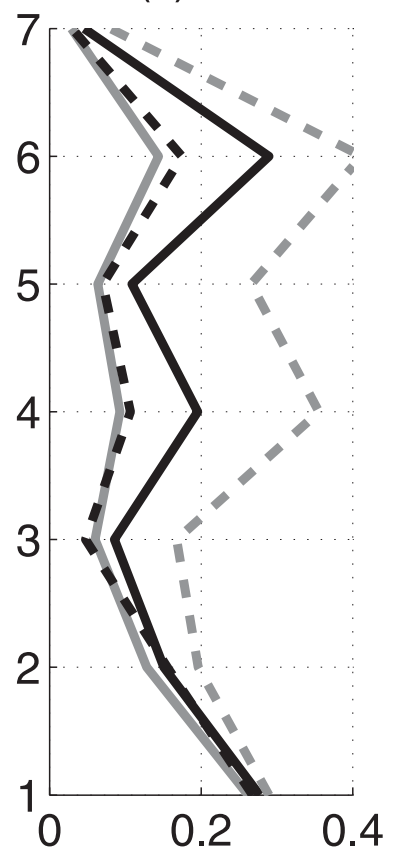

(d) $-Q$

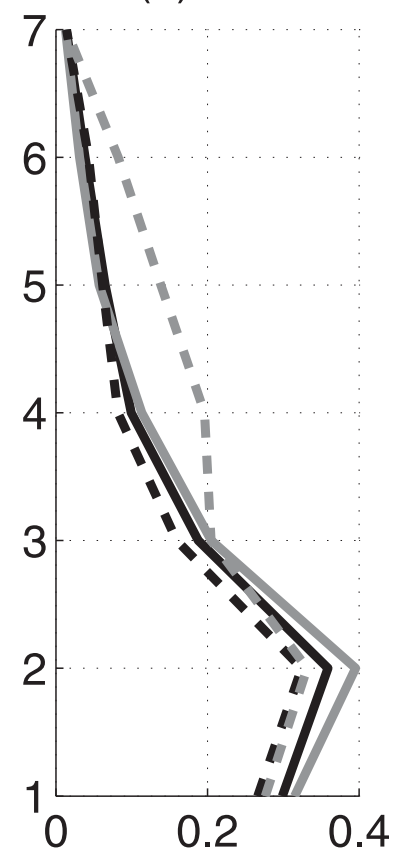

FIG. 5. Absolute mean error (AME) as a function of the vertical model level for (a) the $U$ component of the wind, (b) the $V$ component of the wind, (c) temperature, and (d) specific moisture and for the control (black solid line), parameter estimation (gray solid line), bias correction (black dashed line), and parameter estimation using only low-level moisture observations (gray dashed line) experiments.

ensemble spread based on the information provided by the structure of the analysis error covariance matrix. In the current work, the comparison is carried out in the imperfect model scenario.

The dependence of the initial value of the parameter ensemble spread in the analysis RMSE is evaluated in an experiment that uses the multiplicative and additive inflation. For the multiplicative parameter inflation, the EPES and CCI approaches are used. Eight experiments with different initial parameter ensemble spread were conducted. Figure $7 \mathrm{a}$ shows the time-averaged total RMSE of the analysis as a function of the initial parameter ensemble spread. The CCI approach shows sensitivity to the initial parameter ensemble spread while the EPES approach is almost insensitive to the initial parameter ensemble spread. Moreover, the analysis RMSE obtained with EPES in this experiment is in general as low as the minimum RMSE obtained with CCI approach. Figures $7 \mathrm{~b}$ and $7 \mathrm{c}$ also show the time-averaged estimated parameters as a function of the initial parameter ensemble spread. In the CCI approach, estimated parameter values change as a function of the parameter ensemble spread, while in the EPES approach their estimated values are much less sensitive to the initial parameter ensemble spread. Changes in the estimated parameter values may explain the impact that the initial parameter ensemble spread is producing upon the total analysis RMSE for the CCI approach. In the CCI approach, when the parameter ensemble spread is too small, then optimal parameters are not well estimated (or the convergence is too slow). Furthermore, when the parameter ensemble spread is too large, then parameter values in some ensemble members might be too far from the optimal value, thus degrading the parameter estimation and the performance of some of the ensemble members.

We conclude from these experiments that one important advantage of the EPES approach is that the parameter ensemble spread does not need to be tuned. The tuning of the parameter ensemble spread is computationally expensive, particularly if several parameters are estimated, because the optimal parameter ensemble spread might be (as in the present case) different for each parameter (Zhang et al. 2012). The inflation of the parameter ensemble spread in the EPES approach is directly related to the spread in the state variable ensemble. In these experiments, EPES outperforms CCI for any initial parameter spread. If only multiplicative inflation is used, CCI gives a slightly smaller RMSE than EPES for the optimal initial spread value (not shown).

To evaluate sensitivity of the estimated parameter values to the initial parameter ensemble mean, a set of four experiments which differ in the initial parameter ensemble mean were conducted. The initial parameter 

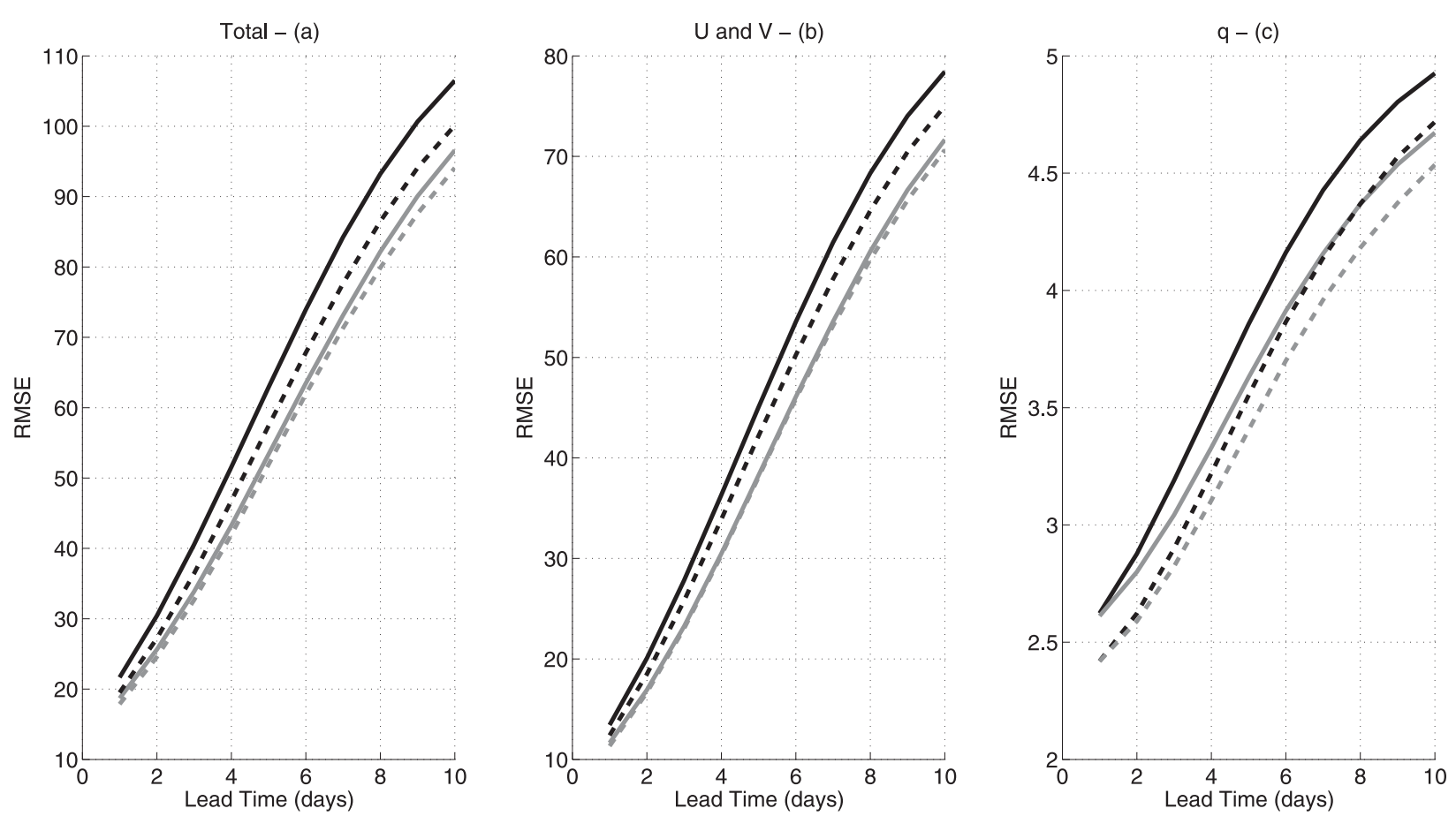

FIG. 6. RMSE of the ensemble mean as a function of forecast lead time and for the control (black solid line), parameter estimation (gray solid line), bias correction (black dashed line), and the combination of parameter estimation and bias correction (gray dashed line) experiments: (a) total RMSE, (b) $U$ and $V$ RMSE, and (c) $q$ RMSE.

ensemble mean is the same for the three convective scheme parameters and is set as $0.2,0.4,0.6$, and 0.8 for each experiment. Figure 8 shows that the estimated parameters in these experiments converge to values that are almost independent of the initial parameter ensemble mean values. We conclude that the estimated parameter values are robust in the sense that they are practically independent of the initial parameter ensemble mean. The instantaneous value of the estimated parameters shows high-frequency temporal variability, and differs from one experiment to the other. This behavior is likely produced by sampling error that is explained by the limited size ensemble used in these experiments as well as because of localization is not used for the parameters (Aksoy 2015). Parameters that produce a high impact upon the model skill (e.g., RHBL) are less sensitive to the effect of sampling noise.

Figure 8 shows that the convergence time depends on the initial parameter value. It can be as long as 300 assimilation cycles. The convergence time of the parameter depends also on the initial parameter ensemble spread; a larger initial parameter ensemble spread can speed up convergence particularly in the cases where the initial parameter value is far from the optimal value. A large convergence time can be a serious limitation for parameter estimation in applications like convective-scale data assimilation in which in order to be useful, the estimated parameters have to converge in a few assimilation cycles. In this case, the impact of techniques like running in place upon parameter estimation should be explored (Yang et al. 2012).

\section{Conclusions}

In this work, a parameter estimation technique based on the LETKF assimilation scheme is evaluated in an imperfect model scenario as a way of accounting for model error within ensemble-based data assimilation. It is found that parameter estimation as a model error treatment technique can improve the analysis via the optimization of the model parameters; on the other hand, the perturbation of the model parameters alone does not produce, in this case, a significant impact on the analysis quality.

The combination of parameter estimation with other model error treatment techniques shows that parameter estimation can produce further improvement in the analysis and forecast quality. In particular, the combination of multiplicative inflation and additive inflation with parameter estimation produces better results than considering either technique alone, because of the partial correction of model error introduced by the optimized parameter values. The improvement produced by parameter estimation is similar to the one produced by 
(a)

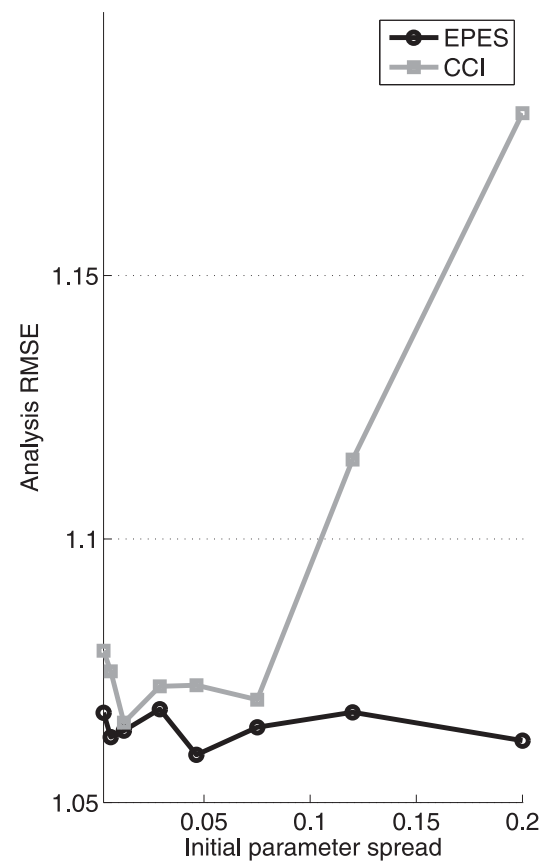

(b)

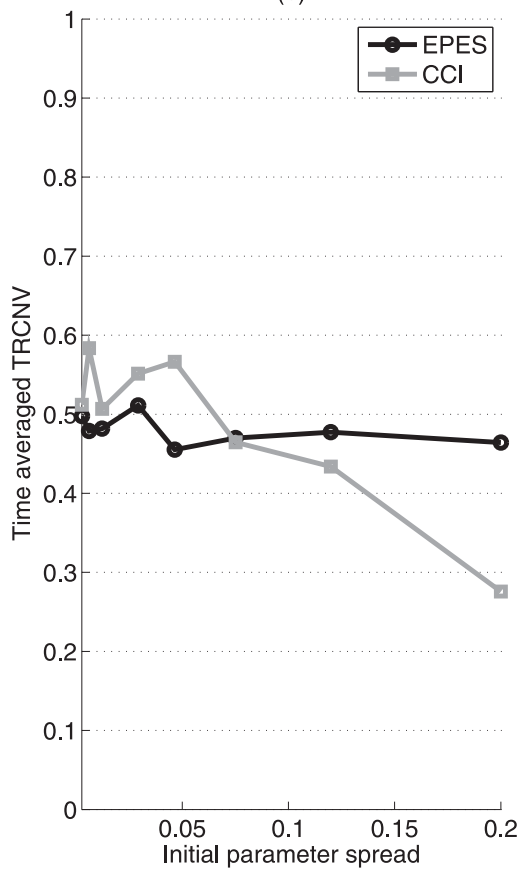

(c)

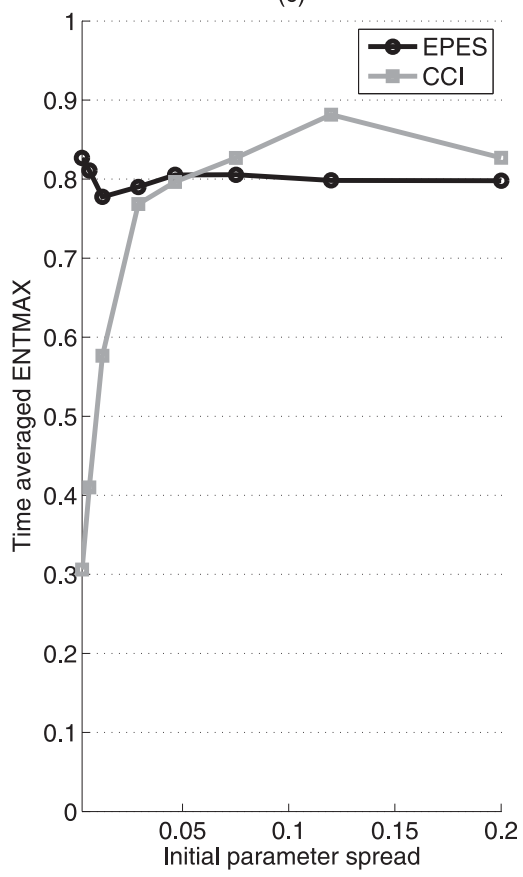

FIG. 7. (a) Time-averaged analysis total RMSE as a function of the initial parameter ensemble spread for the CCI approach (gray line with squares) and the EPES approach (black line with circles). (b) As in (a), but for the time-averaged estimated TRCNV parameter. (c) As in (b), but for the ENTMAX estimated parameter.

a bias correction technique in the experiments with synthetic observations. The advantage in the case of bias correction is that, it introduces a model error correction in a space of the same size as the model state and thus can provide a more consistent correction across different variables in the model. Moreover, several sources of model error can be considered simultaneously with this technique. On the other hand, one potential advantage of parameter estimation is that it can introduce faster timevarying corrections in the time scale associated with the parameterized process. In this work, the correction of model error is performed in a low-dimensional space, a few global parameters are estimated, which can introduce inconsistencies in the impact among different model variables. Because of this low dimensionality only a portion of the model error can be corrected with parameter estimation and the estimation can be seriously affected by the remaining sources of model error. This supports the idea that parameter estimation and bias correction can be combined in order to complement their strengths and mitigate their limitations. In the experiments performed in this work, the combination of parameter estimation and bias correction produces the best analysis. The results obtained in this work suggest that in realistic applications online parameter estimation can be used as a complement to other model error treatment techniques for expert-selected model parameters.
The experiments conducted in this work suggest that the improvement in the analysis RMSE produced by parameter estimation is mainly explained by the optimization of the value of the estimated parameters. The model spread produced by the parameter ensemble perturbations does not have a significant impact upon the analysis. This might be because, in this work, other techniques have also been used in order to improve the ensemble spread for the state variables, thus reducing the impact of the additional spread introduced by the perturbed parameters. Parameter perturbations have also been optimized to produce the best results for parameter estimation but they might be too small to produce a significant impact upon the analysis quality when the parameters are not being estimated and are far from their optimal value.

Consistent with the previously discussed results, we found an important positive impact of parameter estimation in medium-range forecasts. Parameter estimation can improve the RMSE of medium-range forecasts found with the other model error treatment techniques (i.e., additive inflation and bias correction). The improvement in the RMSE is found in all variables except specific moisture, which is degraded in short-term forecasts. However, parameter estimation gives the lowest RMSE for this variable for forecasts with lead times longer than one day. As for the analysis error, the lowest 

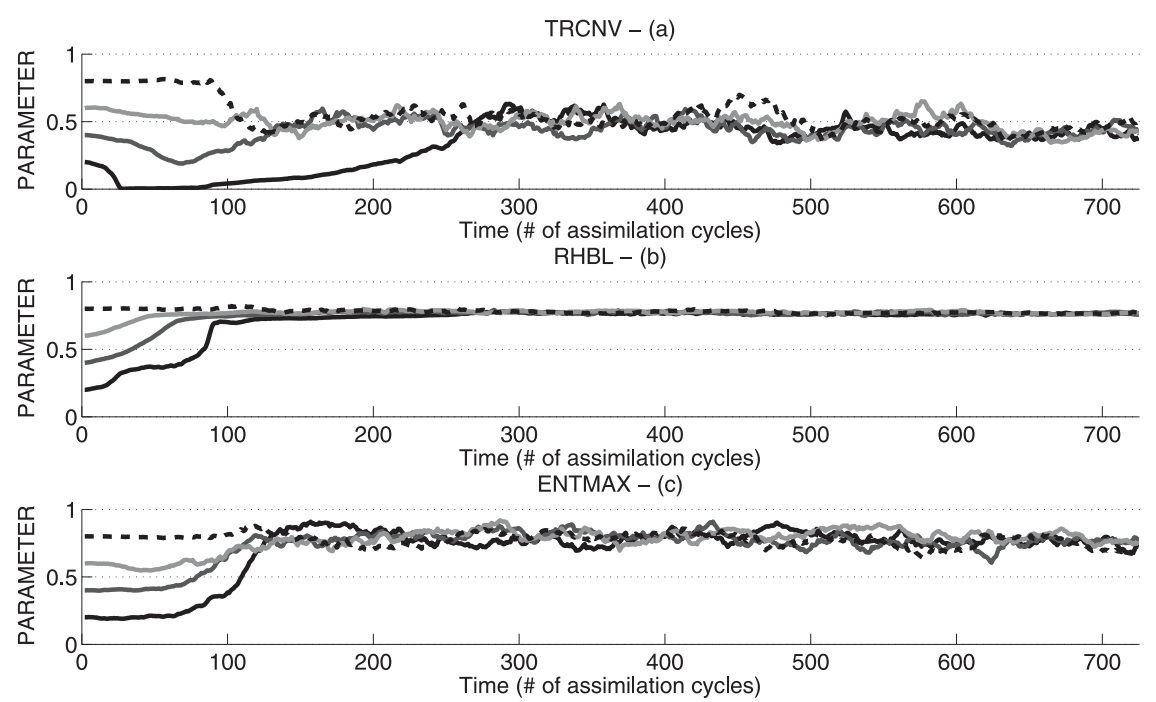

FIG. 8. Time evolution of the parameter ensemble mean for the experiments with an initial parameter ensemble mean of 0.2 (black solid line), 0.4 (dark gray solid line), 0.6 (gray solid line), and 0.8 (black dashed line) and for (a) TRCNV, (b) RHBL, and (c) ENTMAX.

forecast error is achieved by the combination of parameter estimation and bias correction approaches. The lowest forecast error is also achieved, as the lowest analysis error, in the experiment that combines parameter estimation and bias correction techniques.

In the presence of multiple sources of model error, the estimated optimal parameters may differ from the optimal parameters under the assumption of perfect model, say nature or true parameters. Some of the error sources not directly associated with the process represented with the scheme of the parameters to be estimated affect the optimal value of the estimated parameters. This is mainly because model error from different sources may project onto the direction of the model sensitivity to the parameters. In the experiments discussed in this work, the "true" parameter values produce essentially the same analysis RMSE as the imperfect model. On the other hand, the estimated parameters using data assimilation produce a large improvement in the analysis RMSE. This shows that the online estimation of parameters in a data assimilation system can improve the analysis, subject to the parameter values are constrained to an expert-defined range, compared to a system with parameters that are very well offline tuned for a specific situation from observations. The compensation of errors that the parameter optimization produces in an imperfect model scenario may be at the expenses of a worse representation of the process directly associated with the parameter. If the model imperfection is strong, then this error compensation effect can produce unrealistic values in the estimated parameters. To prevent unrealistic parameter values that degrade the forecasts, the parameters must be constrained to an expert-defined range in the data assimilation system. Preliminary experiments in a scenario with more realistic sources of model error, using NCEP-NCAR reanalysis as observations, suggest that the analysis quality may be also improved using parameter estimation. Similar to the results found in this work, the parameters converge to a different value of the default model parameter values for this preliminary experiment with more realistic sources of model error. The detailed results of these experiments will be reported in a follow-up work. Experiments of parameter estimation with real observations and stateof-the-art models have been conducted in Schirber et al. (2013). They found that parameter estimation can improve the short-range forecast or even the model climatology, but that, as in this work, the results might be dependent on the available observations, on the model sensitivity to the parameter, on the magnitude of model error, and on the errors in the observation operator.

It is worth mentioning that parameter estimation is not a way for model improvement, this ultimate goal can only be reached by the improvement of our understanding of the physical processes and to its application to the development of more realistic parameterizations of the unresolved-scale processes (see, e.g., Jakob 2010). Data assimilation schemes in this context can provide an efficient way to estimate the optimal model parameters under the minimum RMSE requirement and so they may be a useful tool to speed up the evaluation and optimization of some model parameterizations under an ever changing model scenario, but the improvement that this technique can produce is strongly constrained by the underlying assumptions made in the design of the parameterization. 
Acknowledgments. This work was partially supported by University of Buenos Aires Grant UBACYT20020100300089; and ANPCyT Grants PICT 2010N1986, PICT 2011 N2452, and PIP 112-20120100414CO. The authors thank Takemasa Miyoshi and Eugenia Kalnay for helpful discussions and also for providing the SPEEDYLETKF code used in the experiments.

The comments provided by three anonymous reviewers were helpful in improving the analysis of the results and the structure of the paper. The authors are also thankful to the editor, Altug Aksoy, for providing fruitful comments.

\section{REFERENCES}

Aksoy, A., 2015: Parameter estimation. Encyclopedia of Atmospheric Sciences, 2nd ed. G. R. North, J. Pyle, and F. Zhang, Eds., Vol. 4, Academic Press, 181-186.

_, F. Zhang, and J. Nielsen-Gammon, 2006a: Ensemble-based simultaneous state and parameter estimation in a two-dimensional sea-breeze model. Mon. Wea. Rev., 134, 2951-2969, doi:10.1175/ MWR3224.1.

$\_, \ldots$, and 2 - 2006b: Ensemble-based simultaneous state and parameter estimation with MM5. Geophys. Res. Lett., 33, L12801, doi:10.1029/2006GL026186.

Anderson, J. L., 2001: An ensemble adjustment Kalman filter for data assimilation. Mon. Wea. Rev., 129, 2884-2903, doi:10.1175/ 1520-0493(2001)129<2884:AEAKFF $>2.0 . C O ; 2$.

_ 2009: Spatially and temporally varying adaptive covariance inflation for ensemble filters. Tellus, 61A, 72-83, doi:10.1111/ j.1600-0870.2008.00361.x.

Annan, J. D., 2005: Parameter estimation using chaotic time series. Tellus, 57A, 709-714, doi:10.1111/j.1600-0870.2005.00143.x.

_ J. C. Hargreaves, N. R. Edwards, and R. Marsh, 2005: Parameter estimation in an intermediate complexity earth system model using an ensemble Kalman filter. Ocean Modell., 8, 135-154, doi:10.1016/j.ocemod.2003.12.004.

Baek, S.-J., B. R. Hunt, E. Kalnay, E. Ott, and I. Szunyogh, 2006: Local ensemble Kalman filtering in the presence of model bias. Tellus, 58A, 293-306, doi:10.1111/j.1600-0870.2006.00178.x.

Bellsky, T., J. Berwald, and L. Mitchell, 2014: Nonglobal parameter estimation using local ensemble Kalman filtering. Mon. Wea. Rev., 142, 2150-2164, doi:10.1175/MWR-D-13-00200.1.

Buizza, R., M. Miller, and T. N. Palmer, 1999: Stochastic representation of model uncertainties in the ECMWF ensemble prediction system. Quart. J. Roy. Meteor. Soc., 125, 2887-2908, doi:10.1002/qj.49712556006.

Cornick, M., B. Hunt, E. Ott, H. Kurtuldu, and M. F. Schatz, 2009: State and parameter estimation of spatiotemporally chaotic systems illustrated by an application to Rayleigh-Bénard convection. Chaos, 19, 013108, doi:10.1063/1.3072780.

Danforth, C., E. Kalnay, and T. Miyoshi, 2007: Estimating and correcting global weather model error. Mon. Wea. Rev., 135, 281-299, doi:10.1175/MWR3289.1.

Dee, D., and A. da Silva, 1998: Data assimilation in the presence of forecast bias. Quart. J. Roy. Meteor. Soc., 124, 269-295, doi:10.1002/ qj. 49712454512.

Fertig, E., B. Hunt, E. Ott, and I. Szunyogh, 2007: Assimilating non-local observations with a local ensemble Kalman filter. Tellus, 59A, 719-730, doi:10.1111/j.1600-0870.2007.00260.x.
Greybush, S. J., E. Kalnay, T. Miyoshi, K. Ide, and B. R. Hunt, 2011: Balance and ensemble Kalman filter localization techniques. Mon. Wea. Rev., 139, 511-522, doi:10.1175/ 2010MWR3328.1.

, R. J. Wilson, R. N. Hoffman, M. J. Hoffman, T. Miyoshi, K. Ide, T. McConnochie, and E. Kalnay, 2012: Ensemble Kalman filter data assimilation of Thermal Emission Spectrometer temperature retrievals into a Mars GCM. J. Geophys. Res., 117, E11008, doi:10.1029/2012JE004097.

Hamill, T., J. S. Whitaker, and C. Snyder, 2001: Distancedependent filtering of background-error covariance estimates in an ensemble Kalman filter. Mon. Wea. Rev., 129, 2776-2790, doi:10.1175/1520-0493(2001)129<2776:DDFOBE >2.0.CO;2.

Houtekamer, P. L., H. L. Mitchell, and X. Deng, 2009: Model error representation in an operational ensemble Kalman filter. Mon. Wea. Rev., 137, 2126-2143, doi:10.1175/2008MWR2737.1.

Hunt, B. R., E. J. Kostelich, and I. Szunyogh, 2007: Efficient data assimilation for spatiotemporal chaos: A local ensemble transform Kalman filter. Physica D, 77, 437-471, doi:10.1016/ j.physd.2006.11.008.

Jakob, C., 2010: Accelerating progress in global atmospheric model development through improved parameterizations. Bull. Amer. Meteor. Soc., 91, 869-875, doi:10.1175/2009BAMS2898.1.

Jazwinski, A. H., 1970: Stochastic and Filtering Theory. Mathematics in Sciences and Engineering Series, Vol. 64, Academic Press, $376 \mathrm{pp}$.

Jung, Y., M. Xue, and G. Zhang, 2010: Simultaneous estimation of microphysical parameters and the atmospheric state using simulated polarimetric radar data and an ensemble Kalman filter in the presence of an observation operator error. Mon. Wea. Rev., 138, 539-562, doi:10.1175/2009MWR2748.1.

Kang, J. S., E. Kalnay, J. Liu, I. Fung, T. Miyoshi, and K. Ide, 2011: "Variable localization" in an ensemble Kalman filter: Application to the carbon cycle data assimilation. J. Geophys. Res., 116, D09110, doi:10.1029/2010JD014673.

Kondrashov, D., C. Sun, and M. Ghil, 2008: Data assimilation for a coupled ocean-atmosphere model. Part II: Parameter estimation. Mon. Wea. Rev., 136, 5062-5076, doi:10.1175/2008MWR2544.1.

Koyama, H., and W. Watanabe, 2010: Reducing forecast errors due to model imperfections using ensemble Kalman filtering. Mon. Wea. Rev., 138, 3316-3332, doi:10.1175/2010MWR3067.1.

Li, H., E. Kalnay, T. Miyoshi, and C. M. Danforth, 2009: Accounting for model errors in ensemble data assimilation. Mon. Wea. Rev., 137, 3407-3419, doi:10.1175/2009MWR2766.1.

Meng, Z., and F. Zhang, 2007: Test of an ensemble Kalman filter for mesoscale and regional-scale data assimilation. Part II: Imperfect model experiments. Mon. Wea. Rev., 135, 14031423, doi:10.1175/MWR3352.1.

Miyoshi, T., 2005: Ensemble Kalman filter experiments with a primitive-equation global model. Ph.D. thesis, University of Maryland, College Park, 197 pp.

- 2011: The Gaussian approach to adaptive covariance inflation and its implementation with the local ensemble transform Kalman filter. Mon. Wea. Rev., 139, 1519-1535, doi:10.1175/ 2010MWR3570.1.

_ , and S. Yamane, 2007: Local ensemble transform Kalman filtering with an AGCM at a T159/L48 resolution. Mon. Wea. Rev., 135, 3841-3861, doi:10.1175/2007MWR1873.1.

,$- \ldots$, and T. Enomoto, 2007: Localizing the error covariance by physical distances within a local ensemble transform Kalman filter (LETKF). SOLA, 3, 89-92, doi:10.2151/sola.2007-023.

Molteni, F., 2003: Atmospheric simulations using a GCM with simplified physical parametrizations. I: Model climatology and 
variability in multi-decadal experiments. Climate Dyn., 20, 175-191, doi:10.1007/s00382-002-0268-2.

Pulido, M., and J. Thuburn, 2006: Gravity wave drag estimation from global analyses using variational data assimlation principles. Part II: Case study. Quart. J. Roy. Meteor. Soc., 132, 1527-1543, doi:10.1256/qj.05.43.

Ruiz, J., M. Pulido, and T. Miyoshi, 2013a: Estimating model parameters with ensemble-based data assimilation: A review. J. Meteor. Soc. Japan, 91, 79-99, doi:10.2151/jmsj.2013-201.

$\_, \ldots$, and $—, 2013 \mathrm{~b}$ : Estimating model parameters with ensemble-based data assimilation: Parameter covariance treatment. J. Meteor. Soc. Japan, 91, 453-469, doi:10.2151/ jmsj.2013-403.

Schirber, S., D. Klocke, R. Pincus, J. Quaas, and J. L. Anderson, 2013: Parameter estimation using data assimilation in an atmospheric general circulation model: From a perfect towards the real world. J. Adv. Model. Earth Syst., 5, 58-70, doi:10.1029/ $2012 \mathrm{MS} 000167$.

Shutts, G., 2005: A kinetic energy backscatter algorithm for use in ensemble prediction systems. Quart. J. Roy. Meteor. Soc., 131, 3079-3102, doi:10.1256/qj.04.106.

Stainforth, D. A., and Coauthors, 2005: Uncertainty in predictions of the climate response to rising levels of greenhouse gases. Nature, 433, 403-406, doi:10.1038/nature03301.
Tong, M., and M. Xue, 2008: Simultaneous estimation of microphysical parameters and atmospheric state with simulated radar data and ensemble square root Kalman filter. Part II: Parameter estimation experiments. Mon. Wea. Rev., 136, 16491668, doi:10.1175/2007MWR2071.1.

Whitaker, J. S., and T. M. Hamill, 2012: Evaluating methods to account for system errors in ensemble data assimilation. Mon. Wea. Rev., 140, 3078-3089, doi:10.1175/MWR-D-11-00276.1.

,,-- X. Wei, Y. Song, and Z. Toth, 2008: Ensemble data assimilation with the NCEP global forecasting system. Mon. Wea. Rev., 136, 463-482, doi:10.1175/2007MWR2018.1.

Wu, X., S. Zhang, Z. Liu, A. Rosati, T. L. Delworth, and Y. Liu, 2012: Impact of geographic dependent parameter optimization on climate estimation and prediction: Simulation with an intermediate coupled model. Mon. Wea. Rev., 140, 3956-3971, doi:10.1175/MWR-D-11-00298.1.

Yang, S.-C., E. Kalnay, and T. Miyoshi, 2012: Improving EnKF spin up for typhoon assimilation and prediction. Wea. Forecasting, 27, 878-897, doi:10.1175/WAF-D-11-00153.1.

Zhang, S., Z. Liu, A. Rosati, and T. Delworth, 2012: A study of enhancive parameter correction with coupled data assimilation for climate estimation and prediction using a simple coupled model. Tellus, 64A, 10963, doi:10.3402/tellusa.v64i0.10963. 